\title{
A NOTE ON AXIOMATIC CHARACTERIZATION OF FIELDS
}

\author{
E. ARTIN AND G. WHAPLES
}

Since publication of our paper, Axiomatic characterization of fields by the product formula for valuations, ${ }^{1}$ we have found that the fields of class field theory can be characterized by somewhat weaker axioms; we can drop the assumption, in Axiom 1 , that $|\alpha|_{\mathfrak{p}}=1$ for all but a finite number of $\mathfrak{p}$, replacing it by the assumption that the product of all valuations converges absolutely to the limit 1 for all $\alpha$.

Our original proof can be adapted to the new axiom with a few modifications, which we shall describe here. In §2, we keep Axiom 1 for reference and introduce:

Axiom 1*. There is a set $\mathfrak{M}$ of prime divisors $\mathfrak{p}$ and a fixed set of valuations ||$_{\mathfrak{p}}$, one for each $\mathfrak{p} \in \mathfrak{M}$, such that, for every $\alpha \neq 0$ of $k$, the product $\Pi_{\mathfrak{p}}|\alpha|_{p}$ converges absolutely to the limit 1 . (That is, the series $\sum_{\mathfrak{p}} \log |\alpha|_{\mathfrak{p}}$ converges absolutely to 0 .)

We must then omit the statement that there are only a finite number of archimedean primes, since this does not follow immediately from $1^{*}$; instead of it, we use the fact that $\sum_{p_{\infty} \rho} \rho\left(p_{\infty}\right)$ and $\sum_{p_{\infty}} \lambda\left(p_{\infty}\right)$ converge absolutely. These quantities are defined on p. 480; the convergence follows from the fact that the product over all $p_{\infty}$ of $|1+1|_{p_{\infty}}$ must converge absolutely. Also, we must temporarily broaden the definition of "parallelotope" so as to permit a parallelotope to be defined by any valuation vector $\mathfrak{a}$ for which $\prod_{\mathfrak{p}}|\mathfrak{a}|_{\mathfrak{p}}$ converges absolutely (rather than restricting $a$ to be an idèle). In the statement of Axiom 2 we must replace "Axiom 1" by "Axiom 1*," Theorem 2, however, is left unchanged, together with Lemmas 4,5 , and 6 , which are needed only to prove it; this theorem shows that the fields of class field theory really satisfy Axiom 1, so that at the end of the whole proof we shall find that Axiom 1 is a consequence of Axioms 1* and 2.

In $\S 3, k$ is assumed to be any field for which Axioms $1^{*}$ and 2 hold. Lemma 8 holds under assumption of Axiom 1*, for our slightly more general parallelotopes; in its proof we have only to note, in case of archimedean primes, that the product $\prod_{p_{\infty}} \mu^{\rho\left(p_{\infty}\right)}$ converges absolutely. In Lemma 9, property 2 must be replaced by:

2*. $|\alpha|_{p_{\infty} \leqq} \leqq B_{p_{\infty}}|y|_{\mathfrak{p}_{\infty}}$, with a set of constants $B_{\mathfrak{p}_{\infty}}$ for which $\prod_{\mathfrak{p}_{\infty}} B_{\mathfrak{p}_{\infty}}$ converges absolutely.

Received by the editors December 9, 1945.

1 Bull. Amer. Math. Soc. vol. 51 (1945) pp. 469-492. 
To prove existence of these constants, let, at each $\mathfrak{p}_{\infty}, M_{p_{\infty}}$ be the maximum of $\left|\alpha_{i}\right|_{p_{\infty}}$ for $i=1 \cdots l$; then $\prod_{p_{\infty}} M_{p_{\infty}}$ converges to a finite limit. Take $B_{p_{\infty}}=M_{p_{\infty}} l^{\lambda\left(p_{\infty}\right)}$; since $\sum_{p_{\infty}} \lambda\left(\mathfrak{p}_{\infty}\right)$ was absolutely convergent, our conclusion follows.

Lemma 10 holds as stated, although the set of $p_{\infty}$ is not now known to be finite. But as soon as we have proved that $n$ is finite, it follows from Theorem 2 that our original Axiom 1 holds, so no further changes are necessary. (The theorems about parallelotopes in $\$ 4$ hold only for parallelotopes defined by ideal elements.)

It is easy to construct an example of a field which satisfies Axiom 1* but does not satisfy Axiom 1 (nor, of course, Axiom 2). Let $k=R(x, z)$ be the set of all rational functions of $x$ and $z$ over the rational field. Let $k_{0}=R(x)$, consider $k$ as the set $k_{0}(z)$ of all rational functions of $z$ with $k_{0}$ as constant field, and denote by $\mathfrak{M}_{0}$ the set of all divisors which are trivial on $k_{0}$. We construct $\mathfrak{M}_{0}$, and define the set of normed valuations, exactly as in the proof of Lemma 6 of our original paper (pp. 477-479). Let $V_{0}(A)=\prod\|A\|_{p_{0}}$ where the product is taken over all $p_{0} \in \mathfrak{M}_{0}$; by Lemma $6, V_{0}(A)=1$ for all $A \in k$.

Now let $x_{1}=x+z, x_{2}=x+2 z, \cdots, x_{i}=x+i z, \cdots$; let $k_{i}=R\left(x_{i}\right)$ and for each $i$ construct the sets $\mathfrak{M}_{i}$ of divisors $p_{i}$ by repeating exactly the above process with $k_{0}$ replaced by $k_{i}$. The products $V_{i}(A)$ are all equal to 1 . These sets $\mathfrak{M}_{i}$ are by no means disjoint; for example one can easily see that the irreducible polynomial $z$ defines the same valuation in each $\mathfrak{M}_{i}$. However, it is unnecessary to explore these duplications in detail; we shall need only the facts that the valuations $p_{i \infty}$ and $p_{j \infty}$ are inequivalent for $i \neq j$, and are not equivalent to any of the finite $p_{p}$. Namely, $x_{i}=x+i z=x_{j}+(i-j) z$ has value 1 at $p_{i \infty}$, but value $q>1$ at all $p_{j \infty}$ with $j \neq i$. And $z$ has value $q>1$ at all $p_{i \infty}$, but has value $\leqq 1$ at all finite $p_{\nu}$.

To construct our example, let $\epsilon_{\nu}(\nu=0,1,2, \cdots)$ be an infinite sequence of positive numbers whose sum is finite. Form the product

$$
\Pi\|A\|_{p_{i}}^{a_{i}}
$$

over all $p_{i} \in \mathfrak{M}_{i}$, all $i$, and in this product unite each set of equivalent valuations into a single valuation. The exponents insure the convergence of the infinite products involved in this step. To show that the whole product is absolutely convergent for each $A \in k$, write $A$ in the form $A=g(x, z) / h(x, z)$ where $g$ and $h$ are polynomials with rational coefficients. If $N$ and $M$ are the maximum degrees in $x$ and $z$, respectively, for both numerator and denominator, then $A$ can be written in the form $g_{i}(z) / h_{i}(z)$, where numerator and denominator are poly- 
nomials in $z$ with coefficients in $k_{i}$, and are of degree at most $N+M$ in $z$. It follows from this that, for fixed $A$, the number of factors of $V_{i}(A)$ which are greater than 1 (or which are less than 1 ) is bounded, and their size is bounded; and this bound is uniform for all $i$. Hence the exponents $\epsilon_{i}$ insure absolute convergence. Finally, we note that our product, applied to $z$, contains an infinity of factors different from 1 .

Taking the product over sets $\mathfrak{M}_{0}$ and $\mathfrak{M}_{1}$ only gives an example in which Axiom 1 is satisfied but Axiom 2 is not; for the field of constants with respect to $\mathfrak{M}_{0} \cup \mathfrak{M}_{1}$ is the rational field $k_{0} \cap k_{1}$.

To get an example of a field possessing a valuation satisfying Axiom 2 , but such that this valuation cannot be contained in any set $\mathfrak{M}$ satisfying Axiom 1, take the $p$-adic closure of either the rational field or any of the fields $k_{0}(z)$ of our original paper, with $p$ any of the divisors of Lemma 6 . Because of Theorem 3, such an $\mathfrak{M}$ cannot exist.

INDIANA UNIVERSITY AND

University of PenNsylvania 